\title{
ECR collective response: the future of criminology and the unsustainability of the status quo for ECRs
}

Sarah Anderson

Shane Horgan

Fiona Jamieson

Cara Jardine Ashley Rogers

This is the accepted manuscript

Anderson, S., Horgan, S., Jamieson, F., Jardine, F. \& Rogers, A. ECR collective response: the future of criminology and the unsustainability of the status quo for ECRs, Criminology and Criminal Justice. Copyright (C) 2020 the authors. DOI: $10.1177 / 1748895820949299$

\section{Information for Users of the Institutional Repository}

Users who receive access to an article through a repository are reminded that the article is protected by copyright and reuse is restricted to non-commercial and no derivative uses. Users may also download and save a local copy of an article accessed in an institutional repository for the user's personal reference. For permission to reuse an article, please follow our Process for Requesting Permission 


\section{ECR Collective Response:}

The Future of Criminology and the Unsustainability of the Status Quo for ECRs

Sarah Anderson (University of the West of Scotland, UK)

Shane Horgan (Edinburgh Napier University, UK)

Fiona Jamieson (University of Edinburgh, UK)

Cara Jardine (University of Strathclyde, UK)

Ashley Rogers (Abertay University, UK)

We were delighted to be asked to respond to Richard Spark's paper. We are encouraged by the themes and issues highlighted, and feel passionately about many of the areas of future research identified in the piece. Indeed, many of the areas of scholarship (such as research with the Global South, practices and experiences of crime and punishment, violence in all its forms, crime and technology, socio-legal research, and political discourses around crime) are areas with which we - as a collective group of early career researchers (ECRs) - are currently engaged, often in collaboration with other ECRs within and outwith the UK. We commend both Prof. Sparks and the ESRC for this important and timely reflection on the direction and possible futures of criminology.

We also highly commend the editors of CCJ who have sought a contribution from a group of academics who are at risk of being marginalised within the discipline 
generally, and from these conversations, specifically. As a collective, we feel the piece raises a number of questions and contingencies related to occupational conditions and culture in UK Higher education. In this brief response, we wish to consider and highlight how certain features of our field may limit the extent to which this exciting criminological future is realised. For brevity, our response will focus on these structural issues. However, we also recognise that the precarity experienced by many ECRs also has a high human cost including, but not limited to: exploitative working conditions; financial insecurity; being unable to make future plans; and rising mental and physical ill health (Jones and Oakley, 2018; Gill, 2009, 2014; UCU, 2019; Thwaites and Pressland, 2017).

As Sparks' notes, inter- and multidisciplinary work is essential if we are to engage fully and critically with the societal issues of our time (e.g. the climate crisis, technologyrelated risks). Arguably, this possible future has already begun to manifest, but this is not without its pitfalls.

Firstly, interdisciplinary and multidisciplinary work involves developing a methodological and philosophical lingua franca with disciplines often far from our own (e.g. climatology, computer science). While desirable, it should be recognised that these developments take time and, in the context of a 'fast-paced' and 'REF ready' research environment, there is little appetite for risky investment. This research context constrains the possible designs and methods available to us (e.g. long-term 
ethnographic fieldwork), restricts the development of fully critical accounts, and risks a criminology that itself contributes to systems of oppression and subjugation. Secondly, silo-bridging conversations may or may not lead to outputs that satisfy both funders and university promotion-track demands, as well as moral and ethical commitments. The extent to which this work is tenable, therefore, both in terms of securing funding or continuing work, for those who risk non-renewal or promotion to permanency, is unclear. The commitment required for such work extends far beyond the generosity or passion of those precariously employed, if employed at all. Even for those on permanent contracts, it far exceeds already unrealistic workload models.

In light of these tensions, it is perhaps unsurprising that PhD students are often at the forefront of multidisciplinary research. The PhD can afford the space to nurture relationships, read, and think; allowing researchers the time required to navigate complex methodological and conceptual barriers and move the discipline forward. However, the extent to which this will remain the case is questionable, and we should exercise caution regarding where and with whom the responsibility to push disciplinary boundaries lies. While there had been some welcome discussion within funding bodies of increasing the number of years of funding available, it appears this will not be the case. While the time to complete becomes shorter, the list of achievements $\mathrm{PhD}$ students are expected to demonstrate grows; to publish in prestigious journals, have real world impact, acquire sufficient teaching experience, and do so while navigating an increasingly competitive and precarious job-market. Thus, focusing on PhD candidates, 
or those equally precarious, to drive forward the establishment of multidisciplinary connections is misguided and unjust.

We can also observe a shift towards larger scale, inter-disciplinary research projects which has implications for the types of employment that ECRs move into. Often, these projects adopt a model of working more familiar to STEM disciplines; a Principal Investigator (the grant holder) leads the project and is supported by one or more postdoctoral researchers. This has coincided with a decreasing number of permanent academic posts, creating a situation whereby many ECRs will become 'serial' postdocs; employed on a succession of fixed-term, temporary, and precarious contracts across different projects. This results in numerous difficulties for ECRs, not limited to: a lack of recognition of the additional time inter-disciplinary research requires (e.g. to learn new skills, methodologies and literatures); difficulties maintaining professional autonomy and an independent identity; and barriers to developmental opportunities (Jones and Oakley, 2018). With regard to progression, post-docs can face a disconnect between their careers and traditional measures of "success", such as producing sole-authored papers. Moving between subjects can leave these ECRs without a disciplinary 'home', and with what appears to be a disjointed narrative to their work history, they may struggle to market themselves in a competitive job market. For inter-disciplinary work to be sustainable, these issues of progression and transitions must be resolved. 
For funders, this model of working has significant implications for the resulting number and quality of research outputs. 'Serial post-docs', for example, often move into new posts with unfinished outputs from previous projects and have limited time to engage in knowledge exchange activities (Jones and Oakley, 2018). It is not always guaranteed that they will continue to be included in any future writing projects and outputs from the original research team. Academia often lacks awareness, kindness and fairness in this respect. This raises serious questions about whether this model of working hampers both academic scholarship and publication, and meaningful engagement with wider audiences. Indeed, a recent survey of nearly 4,000 casualised staff found that $79 \%$ felt that this was not a cost-effective way to fund research, while $96 \%$ felt that more secure employment would support more genuinely innovative scholarship (UCU, 2019).

Funders may also see limited returns when ECRs move from their $\mathrm{PhD}$ to teachingheavy posts. The prevalence of teaching only contracts, which may or may not be permanent, has grown rapidly and over a third of academics involved in teaching are now on a contract of this type (UCU, 2019). These roles often have limited (or no) paid time to do research or to publish from their (often research council funded) $\mathrm{PhD}$ research. Even where ECRs are successful in securing a Lecturer post, staff in combined teaching and research posts spend more than double the time on teaching than on research (reportedly accounting for only $15 \%$ of their time), with increased demands around administration, pastoral care, marking and internal quality assurance (UCU, 2016). Furthermore, the disparity in the realities of such posts in Russell group 
and post-92 universities must be acknowledged. In the latter, teaching loads may be higher and there are greater difficulties securing research funding in criminology, and yet ECRs within many of these institutions are subject to similar expectations (from their own institution and for future career progression) regarding REF and income generation.

Embracing Spark's call to reflect on how criminology might develop, we can imagine one future where the above structural issues remain unaddressed, entrenching and intensifying precarity within the academy. An almost inevitable effect of this will be the limiting of diversity within criminology, as those with the least resources and capital face the highest barriers to an academic career. This is concerning as women and non-white academics are already marginalised in parts of the discipline which attract the most power and prestige, as institutional practices reproduce within the academy the racial and gender discrimination prevalent in neo-liberal societies (Chesney-Lind and Chagnon, 2016). This has clear implications for the nature of knowledge production, but also for future students. In a climate of Black Lives Matter and \#MeToo, students demand and deserve to be taught about matters of race, gender and inequality from diverse faculties.

Yet, we suggest that an alternative future is possible. We persist with hope and desire to be part of the future of criminology, but importantly, we strive for change within and outwith the discipline to ensure that our work in academia is sustainable. While we 
exist here in a collective response to more dominant voices, we ask that ECRs be included more formally and centrally in funding bodies and their visions. Only then can research objectives and expectations be considered fairly alongside the lived realities of a career in academia more generally, and in criminology, more specifically. Revising the visions of funders and institutions in light of the structural difficulties experienced by ECRs offers an opportunity to challenge practices which are to the detriment of all. As Rosalind Gill powerfully argues, precarity is inextricably linked to other damaging and disabling features of the neo-liberal university, such as the intensification of work, a demand to be 'always available', and the stress, exhaustion and overwork that this entails (Gill, 2009). We hope, then, to forge intergenerational solidarities that will allow us to tackle this culture, and in turn avoid reproducing within the discipline the power and intersectional inequalities that frame so much critical work in criminology.

\section{Acknowledgements}

We are very grateful for comments provided by Dr. Ellie Bates on an earlier draft of this response.

\section{Declaration of Conflicting Interest}

The author(s) declared no potential conflicts of interest with respect to the research, authorship, and/or publication of this article. 


\section{References}

Chesney-Lind M \& Chagnon N (2016) Criminology, Gender, and Race: A Case Study of Privilege in the Academy. Feminist Criminology 11(4): 311-333.

Gill R (2009) Breaking the silence: the hidden injuries of the neoliberal university. In Flood R and Gill R (eds) Secrecy and Silence in the Research Process: Feminist Reflections. London: Routledge Routledge, pp.228-244.

Gill R (2014) Academics, Cultural Workers and Critical Labour Studies. Journal of Cultural Economy 7(1): 12-30.

Jones S and Oakley C (2018) The Precarious Postdoc. Durham: Working Knowledge/Hearing the Voice. Available at: http://www.workingknowledgeps.com/wpcontent/uploads/2018/04/WKPS PrecariousPostdoc PDF Interactive.pdf (accessed on 14 August 2019)

Thwaites R and Pressland A (2017) Introduction: Being an early career feminist academic in a changing academy. In Thwaites $\mathrm{R}$ and Pressland A (eds) Being an early career feminist academic. London: Palgrave Macmillan, pp.1-28.

UCU (2016) Workload is an education issue: UCU Workload survey report 2016. Available at: https://www.ucu.org.uk/media/8195/Workload-is-an-education-issue-UCU- 
workload-survey-report-2016/pdf/ucu workloadsurvey fullreport jun16.pdf (accessed on 14 August 2019)

UCU (2019) Counting the costs of casualisation in higher education: Key findings of a survey conducted by the University and College Union. Available at:

https://www.ucu.org.uk/media/10336/Counting-the-costs-of-casualisation-in-higher-

education-Jun-19/pdf/ucu casualisation in HE survey report Jun19.pdf (accessed on 14 August 2019) 